\title{
DECAY ESTIMATES OF THE NON-ISENTROPIC COMPRESSIBLE FLUID MODELS OF KORTEWEG TYPE IN $R^{3 *}$
}

\author{
$\mathrm{XU} \mathrm{ZHANG}^{\dagger}$ AND ZHONG TAN TH $^{\ddagger}$
}

\begin{abstract}
The existence and optimal convergence rates of global-in-time classical solutions to the Cauchy problem for the compressible non-isotropic Navier-Stokes-Korteweg system for small initial perturbation is obtained. The global solution is obtained by combining the local existence and the a priori estimates provided the initial perturbation around a constant state is small enough. The optimal convergence rates are obtained by energy estimates and interpolation inequalities, and without linear decay analysis.
\end{abstract}

Key words. Navier-Stokes equations, Korteweg, optimal decay rates, energy method, Sobolev interpolation.

AMS subject classifications. Primary: 35Q30, 76N10; Secondary: 76D05.

\section{Introduction}

The compressible Navier-Stokes-Korteweg system governs the motions of the compressible viscous capillary fluids. This system was first introduced by Korteweg [4] when he studied the theory of capillarity with diffuse interfaces and later was derived rigorously by Dunn and Serrin [7]. For $x \in \mathbb{R}^{3}, t>0$, this fluid satisfies

$$
\left\{\begin{array}{l}
\partial_{t} \rho+\operatorname{div}(\rho u)=0 \\
\partial_{t}(\rho u)+\operatorname{div}(\rho u \otimes u)+\nabla P=\operatorname{div}[\mathcal{S}+\mathcal{K}] \\
\partial_{t}(\rho \mathcal{E})+\operatorname{div}(\rho u \mathcal{E}+u P)=\operatorname{div}(\alpha \nabla \theta)+\operatorname{div}[(\mathcal{S}+\mathcal{K}) u] .
\end{array}\right.
$$

The capillary tensor $\mathcal{K}$ is expressed as follows:

$$
\mathcal{K}=\frac{k}{2}\left(\Delta \rho^{2}-|\nabla \rho|^{2}\right) \mathbb{I}-k \nabla \rho \otimes \nabla \rho
$$

The stress tensor $\mathcal{S}$ is given by

$$
\mathcal{S}=2 \nu \mathbb{D}(u)+(\lambda \operatorname{div} u) \mathbb{I},
$$

where $\rho(t, x), u(t, x)$, and $\theta(t, x)$ represent the density, the velocity, and absolute temperature. $\mathbb{D}$ denotes the strain tensor, which is a $n \times n$ matrix with $\mathbb{D}_{i j}(u)=$ $\frac{\left(\partial_{i} u_{j}+\partial_{j} u_{i}\right)}{2}$. The pressure $P$ is a function of $\rho$ and $\theta$ with $P_{\rho}(1,1), P_{\theta}(1,1)>0$. $\mathcal{E}$ is the total energy equaling to $\frac{1}{2} u^{2}+C_{v} \theta$ with $C_{v}$ a positive constant. The viscosity coefficients $\lambda, \nu$ satisfy $\lambda>0, \lambda+\frac{2 \nu}{3} \geq 0 . \quad k$ and $\alpha$ represent the capillary coefficient and heat conduction respectively. I denotes the unit matrix.

Recently, a great deal of research has been devoted to many topics of the compressible Navier-Stokes-Korteweg system. Hattori and Li [10, 11] considered the local existence and global existence of smooth solutions for the compressible fluid models of Korteweg type in Sobolev space. Danchin and Desjardins [13] proved existence and

*Received: July 16, 2013; accepted (in revised form): October 9. 2013. Communicated by Benoit Perthame.

†School of Mathematical Sciences, Xiamen University, Fujian 361005, P.R. China (zhangxu889@ yeah.net).

${ }^{\ddagger}$ Corresponding author, School of Mathematical Sciences, Xiamen University, Fujian 361005, P.R. China (ztan85@163.com). 
uniqueness results of suitably smooth solutions for isothermal compressible fluids in critical spaces. Bresch, Desjardins, and Lin [5] and Haspot [3] showed the global existence of weak solutions for the compressible fluid models of Korteweg type. Kotschote [16] proved the local existence of strong solutions for a compressible fluid model of Korteweg type. Wang and Tan $[17,22]$ established the optimal decay rates of global smooth solutions for the compressible fluid models of Korteweg type without any external force. Later, Li extended Wang's result in the case of external force in [18].

Most of these papers considered the isentropic case. So, in this paper, we discuss the global existence and the optimal $L^{2}$ decay rate of solutions for the initial value problem of the three-dimensional non-isentropic compressible Navier-Stokes-Korteweg equation (1.1).

Notation. Throughout this paper, $\nabla^{\ell}$ with an integer $\ell \geq 0$ stands for the usual any spatial derivatives of order $\ell$ and $\nabla^{0} f:=f$. When $\ell<0$ or $\ell$ is not a positive integer, $\nabla^{\ell}$ stands for $\Lambda^{\ell}$ defined by (2.3). We use $\dot{H}^{s}\left(\mathbb{R}^{3}\right), s \in \mathbb{R}$ to denote the homogeneous Sobolev spaces on $\mathbb{R}^{3}$ with norm $\|\cdot\|_{\dot{H}^{s}}$ defined by (2.15), and we use $H^{k}\left(\mathbb{R}^{3}\right)$ to denote the usual Sobolev spaces with norm $\|\cdot\|_{H^{s}}$ and $L^{p}\left(\mathbb{R}^{3}\right), 1 \leq p \leq \infty$ to denote the usual $L^{p}$ spaces with norm $\|\cdot\|_{L^{p}}$. We will employ the notation $a \lesssim b$ to mean that $a \leq C b$ for a universal constant $C>0$ that only depends on the parameters coming from the problem, and the indices $N$ and $s$ coming from the regularity on the data. $\|(\rho, u, q)\|_{H^{k}}^{2}=\|\rho\|_{H^{k}}^{2}+\|u\|_{H^{k}}^{2}+\|q\|_{H^{k}}^{2}$. We also use $C_{0}$ for a positive constant depending additionally on the initial data. In the article, we use $t(n, u, q)$ or $h(n, u, q)$ to represent some function of $(n, u, q)$ during the estimate.

For the global existence and large time behavior of strong solutions, we have the following result.

ThEOREM 1.1. Under the assumption that $\rho_{0}-1 \in H^{N+1},\left(u_{0}(x), \theta_{0}-1\right) \in H^{N}$, $N \geq 3$, and that there exists a constant $\epsilon_{0}$ such that

$$
\left\|\rho_{0}-1\right\|_{H^{4}}+\left\|\left(u_{0}, \theta_{0}-1\right)\right\|_{H^{3}} \leq \epsilon_{0},
$$

then the problem (1.1) admits a unique global solution $(\rho, u, \theta)$ satisfying, for all $t \geq 0$,

$$
\begin{aligned}
& \|(\rho-1)(t)\|_{H^{N+1}}^{2}+\|(u(t), \theta(t)-1)\|_{H^{N}}^{2} \\
& \quad+\int_{0}^{t}\|\nabla \rho(\tau)\|_{H^{N+1}}^{2}+\|\nabla u(\tau)\|_{H^{N}}^{2}+\|\nabla \theta(\tau)\|_{H^{N}}^{2} d \tau \\
& \leq C\left(\left\|\rho_{0}-1\right\|_{H^{N+1}}^{2}+\left\|u_{0}\right\|_{H^{N}}^{2}+\left\|\theta_{0}-1\right\|_{H^{N}}^{2}\right) .
\end{aligned}
$$

If, further, $\left(\rho_{0}-1, \nabla \rho_{0}, u_{0}, \theta_{0}-1\right) \in \dot{H}^{-s}$ for some $s \in[0,3 / 2)$, then, for all $t \geq 0$,

$$
\|\rho(t)-1\|_{\dot{H}^{-s}}^{2}+\|\nabla \rho(t)\|_{\dot{H}^{-s}}^{2}+\|\nabla \rho(t)\|_{\dot{H}^{-s}}^{2}+\|u(t)\|_{\dot{H}^{-s}}^{2}+\|\theta(t)-1\|_{\dot{H}^{-s}}^{2} \leq C_{0},
$$

and for $k=0,1, \cdots, N$, the following decay results hold:

$$
\left\|\nabla^{k}(\rho-1)(t)\right\|_{L^{2}}+\left\|\nabla^{k}(\theta-1)(t)\right\|_{L^{2}}+\left\|\nabla^{k} u(t)\right\|_{L^{2}} \leq C_{0}(1+t)^{-\frac{k+s}{2}} .
$$

COROllary 1.2. Under the assumptions of Theorem 1.1 except that we replace the $\dot{H}^{-s}$ assumption by the assumption that $\rho_{0}, u_{0} \in L^{p}$ for some $p \in(1,2]$, then the following decay results hold:

$\left\|\nabla^{k}(\rho-1)(t)\right\|_{L^{2}}+\left\|\nabla^{k}(\theta-1)(t)\right\|_{L^{2}}+\left\|\nabla^{k} u(t)\right\|_{L^{2}} \leq C_{0}(1+t)^{-\sigma_{p, k}}$ for $k=0,1, \cdots, N$. 
Here the number $\sigma_{p, k}$ is defined by

$$
\sigma_{p, k}:=\frac{3}{2}\left(\frac{1}{p}-\frac{1}{2}\right)+\frac{k}{2}
$$

The rest of this paper is organized as follows. The analytic tools used in this paper will be collected in Section 2. In Section 3, we will do some crucial energy estimates. In Section 4, the estimates of the negative Sobolev norms of the solution are obtained. We will prove Theorem 1.1 in Section 5.

\section{Preliminaries}

Before we present the energy estimates method, we should recall the following useful lemmas which we will use extensively.

Lemma 2.1. If $0 \leq m, \alpha \leq \ell$ and $2 \leq p<\infty$, then we have

$$
\left\|\nabla^{\alpha} f\right\|_{L^{p}} \lesssim\left\|\nabla^{m} f\right\|_{L^{2}}^{1-\theta}\left\|\nabla^{\ell} f\right\|_{L^{2}}^{\theta},
$$

where $0 \leq \theta \leq 1$ and $\alpha$ satisfy

$$
\frac{1}{p}-\frac{\alpha}{3}=\left(\frac{1}{2}-\frac{m}{3}\right)(1-\theta)+\left(\frac{1}{2}-\frac{\ell}{3}\right) \theta
$$

Proof. This can be found in [12, pp. 125, THEOREM] for the case when $\alpha$ is an integer. We only need to prove the fraction case.

Firstly, the $\nabla^{\alpha} f\left(\Lambda^{\alpha} f\right)$ is defined by the inverse Fourier transformation:

$$
\nabla^{s} f(x)=\int_{\mathbb{R}^{3}}|\xi|^{s} \hat{f}(\xi) e^{2 \pi i x \cdot \xi} d \xi
$$

where $\hat{f}$ is the Fourier transform of $f$ and the constant is set to be 1 . Roughly speaking, $\nabla^{\alpha} f$ denotes the $\alpha$ order derivative of $f$. This inequality depends on the following proposition of the homogeneous Sobolev space which can be found in [9, pp. 29],

Proposition 2.2. If $s$ is in $\left[0, \frac{3}{2}\right)$, then the space $\dot{H}^{s}\left(R^{3}\right)$ is continuously embedding in $L^{\frac{2 d}{d-2 s}}\left(R^{3}\right)$. The norm of $\dot{H}^{s}\left(R^{3}\right)$ is defined by

$$
\|u\|_{\dot{H}^{s}\left(R^{3}\right)}^{2}=\int_{R^{3}}|\xi|^{2 s}|\hat{u}(\xi)|^{2} d \xi<\infty .
$$

By the definition of $\nabla^{\alpha} f$, it is easy to check that $\nabla^{\alpha}\left(\nabla^{\gamma} f\right)=\nabla^{\alpha+\gamma} f$, then with the help of Proposition 2.2, we have

$$
\left\|\nabla^{\alpha} f\right\|_{L^{p}} \leq C\left\|\nabla^{\alpha} f\right\|_{\dot{H}^{\gamma}\left(R^{3}\right)}, \text { with } \quad \gamma=3\left(\frac{1}{2}-\frac{1}{p}\right) .
$$

Using the Parseval theorem and Holder's inequality, together with $\left\|\nabla^{s} g\right\|_{L^{2}}=$ $\left\||\xi|^{s} \hat{g}\right\|_{L^{2}}$ and choosing appropriate $m, \ell$ such that

$$
\alpha+\gamma=m(1-\theta)+\ell \theta, \text { where } \alpha+\beta \in[m, \ell] \text { or }[\ell, m], \theta \in[0,1],
$$

we finally have

$$
\left\|\nabla^{\alpha} f\right\|_{\dot{H}^{\gamma}\left(R^{3}\right)}^{2}=\int_{\mathbb{R}^{3}}|\xi|^{2 \gamma}\left(\widehat{\nabla^{\alpha} f}(\xi)\right)^{2} d \xi
$$




$$
\begin{aligned}
& \leq \int_{\mathbb{R}^{3}}|\xi|^{2(\alpha+\gamma)} \hat{f}^{2}(\xi) d \xi \\
& \leq \int_{\mathbb{R}^{3}}\left(|\xi|^{m} \hat{f}(\xi)\right)^{2(1-\theta)}\left(|\xi|^{l} \hat{f}(\xi)\right)^{2 \theta} d \xi \\
& \leq C\left\||\xi|^{m} f\right\|_{L^{2}}^{2(1-\theta)}\left\||\xi|^{\ell} f\right\|_{L^{2}}^{2 \theta} \\
& \leq C\left\|\nabla^{m} f\right\|_{L^{2}}^{2(1-\theta)}\left\|\nabla^{\ell} f\right\|_{L^{2}}^{2 \theta} .
\end{aligned}
$$

This means

$$
\left\|\nabla^{\alpha} f\right\|_{L^{p}} \leq C\left\|\nabla^{m} f\right\|_{L^{2}}^{1-\theta}\left\|\nabla^{\ell} f\right\|_{L^{2}}^{\theta},
$$

where $\alpha, \theta, m$, and $\ell$ satisfy

$$
\alpha+3\left(\frac{1}{2}-\frac{1}{p}\right)=m(1-\theta)+\ell \theta .
$$

This inequality is correct for the case $p=\infty$, and one may check the [14] for detailed derivation.

Next is the Gagliardo-Nirenberg interpolation inequality.

Lemma 2.3. For $m=|\alpha|$, and $j=0,1, \cdots, m-1,1 \leq p, 1 \leq q$,

$$
\left\|D^{j} u\right\|_{L^{r}\left(\mathbb{R}^{3}\right)} \leq C\left\{\left\|D^{m} u\right\|_{L^{p}\left(\mathbb{R}^{3}\right)}\right\}^{a}\left\{\|u\|_{L^{q}\left(\mathbb{R}^{3}\right)}\right\}^{1-a},
$$

here

$$
\frac{j}{n}-\frac{1}{r}=a\left(\frac{m}{n}-\frac{1}{p}\right)+(1-a)\left(0-\frac{1}{q}\right)
$$

and

$$
\left\{\begin{array}{l}
a \in\left[\frac{j}{m}, 1\right], \text { with } a \neq 1 \text { if } 1<p<\infty \text { and } m-j-\frac{n}{p} \in\{0\} \bigcup N ; \\
\text { If } j=0, p m<n, q=\infty \text {, then } u \rightarrow 0(|x| \rightarrow \infty) \text {, or }\|u\|_{L^{w}\left(\mathbb{R}^{3}\right)}<\infty, w>0 .
\end{array}\right.
$$

Then in order to establish the Negative Sobolev estimate, we should review the following necessary lemmas related to the negative Sobolev norm.

The operator $\Lambda^{s}\left(\nabla^{s}\right)$ in $R^{n}$ for $s \in \mathbb{R}$ is defined by

$$
\Lambda^{s} g(x)=\int_{\mathbb{R}^{n}}|\xi|^{s} \hat{g}(\xi) e^{2 \pi i x \cdot \xi} d \xi
$$

where $\hat{g}$ is the Fourier transform of $g$ and the constant has been set to 1 . When the $s$ is negative, we rewrite $s$ as $-\alpha$ with $\alpha>0 . \Lambda^{-\alpha}$ is the usual Riesz potential operator. There exists another definition for the Riesz potential operator. We will show these definitions are equal.

Lemma 2.4. The Riesz potential operator $I_{\alpha} f$ in $R^{n}$ can be defined as

$$
\left(I_{\alpha} f\right)(x)=\frac{1}{C_{n, \alpha}} \int_{\mathbb{R}^{n}} \frac{f(y)}{|x-y|^{n-a}} d y \text { with } C_{n, \alpha}=\frac{\Pi^{\frac{n}{2}} 2^{\alpha} \Gamma\left(\frac{\alpha}{2}\right)}{\Gamma\left(\frac{n-\alpha}{2}\right)},
$$


or if we set the constant to be 1 ,

$$
\Lambda^{-\alpha} f(x)=\int_{\mathbb{R}^{n}}|\xi|^{-\alpha} \hat{f}(\xi) e^{2 \pi i x \cdot \xi} d \xi
$$

then (2.10) is equivalent to (2.11).

Proof. From (2.10), if we set $K_{\alpha}(x)=\frac{1}{C_{n, \alpha}|x|^{n-\alpha}}$, we can rewrite $I_{\alpha} f(x)$ as

$$
\left(I_{\alpha} f\right)(x)=K * f .
$$

Taking Fourier transform to $\left(I_{\alpha} f\right)(x)$, we have

$$
\left(\hat{I_{\alpha}} f\right)=\hat{K_{\alpha}} \hat{f} .
$$

To compute $\hat{K}_{\alpha}$, we recall the following theorem in [9, pp. 23],

Proposition 2.5. If $\sigma \in(0, d)$, then $\mathcal{F}\left(|x|^{-\sigma}\right)(\xi)=c_{d, \delta}|\xi|^{\sigma-d}$ for some constant $c_{d, \sigma}$ depending only on $d$ and $s$, where $d$ denotes the dimension of space.

Set $\sigma=n-\alpha$, then we obtain

$$
\left(\hat{I_{\alpha} f}\right)=\frac{1}{(2 \pi)^{\alpha}}|\xi|^{-\alpha} \hat{f}(\xi)
$$

Taking the Fourier inverse transform and setting the constant to be 1, we have

$$
I_{\alpha} f=\int_{\mathbb{R}^{n}}|\xi|^{-\alpha} \hat{f}(\xi) e^{2 \pi i x \cdot \xi} d \xi
$$

We can define the homogeneous Sobolev space $\dot{H}^{s}$ of $g$ with the following norm:

$$
\|g\|_{\dot{H}^{s}}:=\left\|\Lambda^{s} g\right\|_{L^{2}}=\left\||\xi|^{s} \hat{g}\right\|_{L^{2}} .
$$

The index $s$ can be any non-positive real number. However, for convenience, we will change the index to be "-s" with $s \geq 0$, in this case. We will employ the following special Sobolev interpolation that related the negative index $s$ :

Lemma 2.6. Let $s \geq 0$ and $l \geq 0$, then we have

$$
\left\|\nabla^{l} g\right\|_{L^{2}} \leq\left\|\nabla^{l+1} g\right\|_{L^{2}}^{1-\theta}\|g\|_{\dot{H}^{-s}}^{\theta}, \quad \text { where } \quad \theta=\frac{1}{l+s+1} .
$$

Proof. By the Parseval theorem, the definition of (2.15) and Hölder's inequality, we have

$$
\left\|\nabla^{l} g\right\|_{L^{2}}=\left\||\xi|^{l} \hat{g}\right\|_{L^{2}} \leq\left\||\xi|^{l+1} \hat{g}\right\|_{L^{2}}^{1-\theta}\left\||\xi|^{-s} \hat{g}\right\|_{L^{2}}^{\theta}=\left\|\nabla^{l+1} g\right\|_{L^{2}}^{1-\theta}\|g\|_{\dot{H}^{-s}}^{\theta}
$$

Lemma 2.7. Assume that $\|(\rho, u)\|_{H^{3}} \leq c_{0} \leq 1$, and Let $f(\rho)$ be a smooth function of $\rho$. Then for any integer $k \geq 1$ we have

$$
\left\|\nabla^{l} f(\rho) \cdot \nabla^{k-l} u\right\|_{L^{2}} \lesssim c_{0}\left\|\nabla^{k}(\rho, u)\right\|_{L^{2}} .
$$


Proof. For $1 \leq k, 1 \leq l \leq k$, using the Leibniz formula for $\sum_{i=1}^{n} \gamma_{i}=l$ we have $\nabla^{l} f(n) \cdot \nabla^{k-l} u=$ a sum of products $f^{\gamma_{1}, \ldots, \gamma_{n}}(\rho) \nabla^{\gamma_{1}} \rho \cdots \nabla^{\gamma_{n}} \rho \nabla^{k-l} u$, with $\gamma_{i} \geq 1$.

By the Sobolev interpolation inequality, we have $\|\rho\|_{L^{\infty}} \leq\|\rho\|_{H^{3}} \leq c_{0} \leq 1$. So, we have

$$
\left|f^{\gamma_{1}, \ldots, \gamma_{n}}(\rho)\right| \leq C \text {, C depends only on function } f \text {. }
$$

Using the Hölder inequality and the Gagliardo-Nirenberg interpolation inequality, we have

$$
\begin{aligned}
\left\|\nabla^{l} f(n) \nabla^{k-l} u\right\|_{L^{2}} & \lesssim\left\|\nabla^{\gamma_{1}} \rho\right\|_{\frac{2 k}{\gamma_{1}}}\left\|\nabla^{\gamma_{2}} \rho\right\|_{\frac{2 k}{\gamma_{2}}} \cdot\left\|\nabla^{\gamma_{n}} \rho\right\|_{\frac{2 k}{\gamma_{n}}}\left\|\nabla^{k-l} u\right\|_{\frac{2 k}{k-l}} \\
& \lesssim\|\nabla \rho\|_{L^{3}}{ }^{1-\frac{\gamma_{1}}{k}}\left\|\nabla^{k} \rho\right\|_{L^{2}}{ }^{\frac{\gamma_{1}}{k}} \cdots\|\nabla \rho\|_{L^{3}}{ }^{1-\frac{\gamma_{n}}{k}} \\
& \quad \times\left\|\nabla^{k} \rho\right\|_{L^{2}}{ }^{\frac{\gamma_{n}}{k}}\|\nabla u\|_{L^{3}}{ }^{1-\frac{k-l}{k}}\left\|\nabla^{k} u\right\|_{L^{2}}{ }^{\frac{k-l}{k}} \\
& \lesssim\|\nabla(\rho, u)\|_{L^{3}}{ }^{n-1}\left\|\nabla^{k}(\rho, u)\right\|_{L^{2}} \\
& \lesssim c_{0}\left\|\nabla^{k}(\rho, u)\right\|_{L^{2}} .
\end{aligned}
$$

\section{3. $L^{2}$ energy estimates}

Denote $n=\rho-1, u=u, q=\theta-1, f(n)=\frac{n}{n+1}, g(n, q)=\frac{p_{n}(n+1, q+1)}{n+1}-1, h(n, q)=$ $\frac{p_{q}(n+1, q+1)}{n+1}-1$, and $B(n, q)=\frac{p(n+1, q+1)}{n+1}-1$. Without loss of generality, we assume $P_{p}(1,1)=P_{\theta}(1,1)=C_{v}=k=\lambda=1$. We can write the equation (1.1) as

$$
\left\{\begin{array}{l}
\partial_{t} n+\text { divu }=g_{1}, \\
\partial_{t} u-\mu \Delta u-(\mu+\lambda) \nabla \text { divu }+\nabla n+\nabla q-\nabla \Delta n=g_{2}, \\
\partial_{t} q-\Delta q+\nabla \cdot u=g_{3}
\end{array}\right.
$$

where

$$
\left\{\begin{array}{l}
g_{1}=-\operatorname{div}(n u) \\
g_{2}=-u \nabla u-f(n)(\mu \Delta u+(\mu+\lambda) \nabla \operatorname{div} u)-g(n, q) \nabla n-h(n, q) \nabla q, \\
g_{3}=-u \nabla q+f(n) \Delta q-B(n, q) \nabla u+\frac{1}{n+1}\left[2 \mu D(u): D(u)+\nu(\nabla u)^{2}\right]+\frac{\operatorname{div} \mathcal{K} u}{n+1} .
\end{array}\right.
$$

In this section, we will derive the a priori energy estimates for the equivalent system (1.1). Hence we assume a priori that for sufficiently small $\epsilon>0$,

$$
\sqrt{\mathcal{E}_{0}^{3}(t)}=\|n(t)\|_{H^{4}}+\|u(t)\|_{H^{3}}+\|q(t)\|_{H^{3}} \leq \epsilon .
$$

Hence, for any $k \geq 1$, we immediately have

$$
\begin{aligned}
& |f(n)|,|g(n, q)|,|h(n, q)|,|B(n, q)| \leq C|n||q|, \\
& \left|f^{(k)}(n)\right|,\left|g^{(k)}(n, q)\right|,\left|h^{(k)}(n, q)\right|,\left|B^{(k)}(n, q)\right| \leq C .
\end{aligned}
$$

Lemma 3.1. Under the assumption (3.3), for $k=0,1, \cdots, N$, we have

$$
\frac{1}{2} \frac{d}{d t} \int_{\mathbb{R}^{3}}\left|\nabla^{k} n\right|^{2} d x+\int_{\mathbb{R}^{3}} \nabla^{k} \operatorname{div} u \cdot \nabla^{k} n d x \lesssim \sqrt{\mathcal{E}_{0}^{3}}\left(\left\|\nabla^{k+1} n\right\|_{L^{2}}^{2}+\left\|\nabla^{k+1} u\right\|_{L^{2}}^{2}\right) .
$$



$\mathbb{R}^{3}$,

Proof. Applying $\nabla^{k}$ to $(3.1)_{1}$, multiplying by $\nabla^{k} n$ and integrating by part over

$$
\begin{aligned}
& \frac{1}{2} \frac{d}{d t} \int_{\mathbb{R}^{3}}\left|\nabla^{k} n\right|^{2} d x+\int_{\mathbb{R}^{3}} \nabla^{k} \operatorname{div} u \cdot \nabla^{k} n d x \lesssim \int_{\mathbb{R}^{3}} \nabla^{k} \operatorname{div}(n u) \cdot \nabla^{k} n d x \\
\lesssim & \left\|\nabla^{k} n\right\|_{L^{6}}\left\|\nabla^{k+1}(n u)\right\|_{L^{\frac{6}{5}}} \lesssim\left\|\nabla^{k+1} n\right\|_{L^{2}}\left\|\nabla^{k+1}(n u)\right\|_{L^{\frac{6}{5}}} .
\end{aligned}
$$

To estimate the $\left\|\nabla^{k+1}(n u)\right\|_{L^{\frac{6}{5}}}$ term,

$$
\begin{aligned}
\left\|\nabla^{k+1}(n u)\right\|_{L^{\frac{6}{5}}} & =C_{l} \sum_{l=0}^{k+1}\left\|\nabla^{l} n \nabla^{k+1-l} u\right\|_{L^{\frac{6}{5}}} \\
& =C_{l} \sum_{l=0}^{\left[\frac{k+1}{2}\right]}\left\|\nabla^{l} n \nabla^{k+1-l} u\right\|_{L^{\frac{6}{5}}}+C_{l} \sum_{l=\left[\frac{k+1}{2}\right]+1}^{k+1}\left\|\nabla^{l} u \nabla^{k+1-l} u\right\|_{L^{\frac{6}{5}}} \\
& =W_{1}+W_{2} .
\end{aligned}
$$

For $l \leq\left[\frac{k+1}{2}\right]$, together with the Sobolev interpolation of Lemma 2.1, we have

$$
\begin{aligned}
\left\|W_{1}\right\|_{L^{\frac{6}{5}}} & \leq C \sum_{l=0}^{\left[\frac{k+1}{2}\right]}\left\|\nabla^{l} n \nabla^{k+1-l} u\right\|_{L^{\frac{6}{5}}} \leq\left\|\nabla^{l} n\right\|_{L^{3}}\left\|\nabla^{k+1-l} u\right\|_{L^{2}} \\
& \lesssim\left\|\nabla^{\alpha} n\right\|_{L^{2}}^{\theta}\left\|\nabla^{k+1} n\right\|\left\|_{L^{2}}^{1-\theta}\right\| u\left\|_{L^{2}}^{1-\theta}\right\| \nabla^{k+1} u \|_{L^{2}}^{\theta} \\
& \lesssim \sqrt{\mathcal{E}_{0}^{3}}\left\|\nabla^{k+1} u\right\|_{L^{2}}^{\theta}\left\|\nabla^{k+1} n\right\|_{L^{2}}^{1-\theta} .
\end{aligned}
$$

Here $\alpha, \theta$ satisfy

$$
\left\{\begin{array}{l}
\frac{l}{3}-\frac{1}{3}=\left(\frac{\alpha}{3}-\frac{1}{2}\right) \theta+\left(\frac{k+1}{3}-\frac{1}{2}\right)(1-\theta), \\
\frac{k+1-l}{3}-\frac{1}{2}=\left(\frac{0}{3}-\frac{1}{2}\right)(1-\theta)+\left(\frac{k+1}{3}-\frac{1}{2}\right) \theta .
\end{array}\right.
$$

From (3.8),

$$
\theta=\frac{k+1-l}{k+1}, \alpha=\frac{k+1}{2(k+1-l)} .
$$

So, $0<\theta<1, \alpha \in\left[\frac{1}{2}, 1\right)$.

When $l \geq\left[\frac{k+1}{2}\right]+1$, in the same fashion, the following estimates are obtained:

$$
\left\|W_{2}\right\|_{L^{\frac{6}{5}}} \leq \sqrt{\mathcal{E}_{0}^{3}}\left\|\nabla^{k+1} u\right\|_{L^{2}}^{\theta}\left\|\nabla^{k+1} n\right\|_{L^{2}}^{1-\theta} .
$$

So, from (3.7) and (3.9), we obtain

$$
\left\|\nabla^{k+1}(n u)\right\|_{L^{\frac{6}{5}}} \lesssim \sqrt{\mathcal{E}_{0}^{3}}\left(\left\|\nabla^{k+1} n\right\|_{L^{2}}+\left\|\nabla^{k+1} u\right\|_{L^{2}}\right) .
$$

From (3.6) and (3.10), we conclude the proof of Lemma 3.1.

Lemma 3.2. Under the assumption (3.3), for $k=0,1, \cdots, N$, there exist a positive constant $C$ satisfying

$$
\frac{1}{2} \frac{d}{d t} \int_{\mathbb{R}^{3}}\left|\nabla^{k} u\right|^{2}+\kappa\left|\nabla^{k} \nabla n\right|^{2} d x+C \int_{\mathbb{R}^{3}}\left|\nabla \nabla^{k} u\right|^{2} d x
$$




$$
\begin{gathered}
+\int_{\mathbb{R}^{3}} \nabla^{k} u \cdot \nabla^{k} \nabla n d x+\int_{\mathbb{R}^{3}} \nabla^{k} u \cdot \nabla^{k} \nabla q d x \\
\lesssim \sqrt{\mathcal{E}_{0}^{3}}\left(\left\|\nabla^{k+1} n\right\|_{L^{2}}^{2}+\left\|\nabla^{k+2} n\right\|_{L^{2}}^{2}+\left\|\nabla^{k+1} q\right\|_{L^{2}}^{2}+\left\|\nabla^{k+1} u\right\|_{L^{2}}^{2}\right) .
\end{gathered}
$$

Proof. Applying $\nabla^{k}$ to $(3.1)_{2}$, multiplying $\nabla^{k} u$ and integrating by parts over $\mathbb{R}^{3}$,

$$
\begin{aligned}
& \frac{1}{2} \frac{d}{d t} \int_{\mathbb{R}^{3}}\left(\nabla^{k} u\right)^{2} d x+\mu \int_{\mathbb{R}^{3}}\left(\nabla \nabla^{k} u\right)^{2} d x+(\mu+\lambda) \int_{\mathbb{R}^{3}}\left(\nabla^{k} \operatorname{div} u\right)^{2} \\
& \quad+\int_{\mathbb{R}^{3}} \nabla^{k} u \cdot \nabla^{k} \nabla n d x d x+\int_{\mathbb{R}^{3}} \nabla^{k} u \cdot \nabla^{k} \nabla q d x d x-\int_{\mathbb{R}^{3}} \nabla \Delta \nabla^{k} n \cdot \nabla^{k} u d x \\
& =-\int_{\mathbb{R}^{3}} \nabla^{k}(u \cdot \nabla u) \cdot \nabla^{k} u d x+\int_{\mathbb{R}^{3}} \nabla^{k}[f(n)(\mu \Delta u+(\mu+\lambda) \nabla d i v u)] \cdot \nabla^{k} u d x \\
& \quad \quad-\int_{\mathbb{R}^{3}} \nabla^{k}(g(n, q) \nabla n) \cdot \nabla^{k} u d x+\int_{\mathbb{R}^{3}} \nabla^{k}(h(n, q) \nabla q) \cdot \nabla^{k} u d x \\
& =I_{1}+I_{2}+I_{3}+I_{4} .
\end{aligned}
$$

We treat the $-\int_{\mathbb{R}^{3}} \nabla \Delta \nabla^{k} n \nabla^{k} u d x$ term first.

$$
\begin{aligned}
& -\int_{\mathbb{R}^{3}} \nabla \Delta \nabla^{k} n \cdot \nabla^{k} u d x=\int_{\mathbb{R}^{3}} \Delta \nabla^{k} n \cdot \nabla^{k} \operatorname{div} u d x \\
= & \int_{\mathbb{R}^{3}} \Delta \nabla^{k} n \cdot \nabla^{k}\left(-n_{t}-\operatorname{div}(n u)\right) d x \\
= & \frac{1}{2} \frac{d}{d t} \int_{\mathbb{R}^{3}}\left(\nabla^{k} \nabla n\right)^{2} d x-\int_{\mathbb{R}^{3}} \Delta \nabla^{k} n \cdot \nabla^{k} \operatorname{div}(n u) d x \\
= & \frac{1}{2} \frac{d}{d t} \int_{\mathbb{R}^{3}}\left(\nabla^{k} \nabla n\right)^{2} d x-I_{5} .
\end{aligned}
$$

Next, we treat $I_{i}, i=1, \cdots, 4$. We now estimate the term $I_{1}$. Employing the Leibniz formula and the Hölder and Sobolev inequalities, we obtain

$$
\begin{aligned}
I_{1} & =-\int_{\mathbb{R}^{3}} \nabla^{k}(u \cdot \nabla u) \cdot \nabla^{k} u d x=-\sum_{0 \leq \ell \leq k} C_{k}^{\ell} \int_{\mathbb{R}^{3}}\left(\nabla^{\ell} u \cdot \nabla \nabla^{k-\ell} u\right) \cdot \nabla^{k} u d x \\
& \lesssim \sum_{0 \leq \ell \leq k}\left\|\nabla^{\ell} u \cdot \nabla^{k-\ell+1} u\right\|_{L^{\frac{6}{5}}}\left\|\nabla^{k} u\right\|_{L^{6}} \\
& \lesssim \sum_{0 \leq \ell \leq k}\left\|\nabla^{\ell} u \cdot \nabla^{k-\ell+1} u\right\|_{L^{\frac{6}{5}}}\left\|\nabla^{k+1} u\right\|_{L^{2}} .
\end{aligned}
$$

If $\ell \leq\left[\frac{k}{2}\right]$, by Hölder's inequality and Lemma 2.1 we have

$$
\begin{aligned}
\left\|\nabla^{\ell} u \cdot \nabla^{k-\ell+1} u\right\|_{L^{\frac{6}{5}}} & \lesssim\left\|\nabla^{\ell} u\right\|_{L^{3}}\left\|\nabla^{k-\ell+1} u\right\|_{L^{2}} \\
& \lesssim\left\|\nabla^{\alpha} u\right\|_{L^{2}}^{1-\frac{\ell}{k+1}}\left\|\nabla^{k+1} u\right\|_{L^{2}}^{\frac{\ell}{k+1}}\|u\|_{L^{2}}^{\frac{\ell}{k+1}}\left\|\nabla^{k+1} u\right\|_{L^{2}}^{1-\frac{\ell}{k+1}} \\
& \lesssim \sqrt{\mathcal{E}_{0}^{3}}\left\|\nabla^{k+1} u\right\|_{L^{2}},
\end{aligned}
$$

where $\alpha$ is defined by

$$
\begin{gathered}
\frac{\ell}{3}-\frac{1}{3}=\left(\frac{\alpha}{3}-\frac{1}{2}\right) \times\left(1-\frac{\ell}{k+1}\right)+\left(\frac{k+1}{3}-\frac{1}{2}\right) \times \frac{\ell}{k+1} \\
\Longrightarrow \alpha=\frac{k+1}{2(k+1-\ell)} \in\left[\frac{1}{2}, 1\right) \text { because } \ell \leq \frac{k}{2} .
\end{gathered}
$$


If $\ell \geq\left[\frac{k}{2}\right]+1$, by Hölder's inequality and Lemma 2.1 again we have

$$
\begin{aligned}
\left\|\nabla^{\ell} u \cdot \nabla^{k-\ell+1} u\right\|_{L^{\frac{6}{5}}} & \lesssim\left\|\nabla^{\ell} u\right\|_{L^{2}}\left\|\nabla^{k-\ell+1} u\right\|_{L^{3}} \\
& \lesssim\|u\|_{L^{2}}^{1-\frac{\ell}{k+1}}\left\|\nabla^{k+1} u\right\|_{L^{2}}^{\frac{\ell}{k+1}}\left\|\nabla^{\alpha} u\right\|_{L^{2}}^{\frac{\ell}{k+1}}\left\|\nabla^{k+1} u\right\|_{L^{2}}^{1-\frac{\ell}{k+1}} \\
& \lesssim \sqrt{\mathcal{E}_{0}^{3}}\left\|\nabla^{k+1} u\right\|_{L^{2}}
\end{aligned}
$$

where $\alpha$ is defined by

$$
\begin{gathered}
\frac{k-\ell+1}{3}-\frac{1}{3}=\left(\frac{\alpha}{3}-\frac{1}{2}\right) \times \frac{\ell}{k+1}+\left(\frac{k+1}{3}-\frac{1}{2}\right) \times\left(1-\frac{\ell}{k+1}\right) \\
\Longrightarrow \alpha=\frac{k+1}{2 \ell} \in\left(\frac{1}{2}, 1\right] \text { because } \ell \geq \frac{k+1}{2} .
\end{gathered}
$$

In light of (3.15) and (3.17), we deduce from (3.14) that

$$
I_{1} \lesssim \sqrt{\mathcal{E}_{0}^{3}}\left\|\nabla^{k+1} u\right\|_{L^{2}}^{2}
$$

Next, we estimate the term $I_{2}$. We do the approximation to simplify the presentations as

$$
I_{2}:=\int_{\mathbb{R}^{3}}-\nabla^{k}[f(n)(\mu \triangle u+(\mu+\lambda) \nabla \operatorname{div} u)] \nabla^{k} u d x \approx-\int_{\mathbb{R}^{3}} \nabla^{k}\left(f(n) \nabla^{2} u\right) \cdot \nabla^{k} u d x .
$$

Because $k \geq 1$, we can integrate by parts to have

$$
\begin{aligned}
I_{2} & \approx \int_{\mathbb{R}^{3}} \nabla^{k-1}\left(f(n) \nabla^{2} u\right) \cdot \nabla^{k+1} u d x \\
& \lesssim \sum_{0 \leq l \leq k-1} \int_{\mathbb{R}^{3}} \nabla^{l} f(n) \cdot \nabla^{k-l+1} u \cdot \nabla^{k+1} u d x \\
& \lesssim \sum_{0 \leq l \leq k-1}\left\|\nabla^{l} f(n) \nabla^{k-l+1} u\right\|_{L^{2}}\left\|\nabla^{k+1} u\right\|_{L^{2}} .
\end{aligned}
$$

If $l=0$, because $f(n)=\frac{n}{n+1}$,

$$
\left\|f(n) \nabla^{k+1} u\right\|_{L^{2}} \lesssim \sqrt{\mathcal{E}_{0}^{3}}\left\|\nabla^{k+1} u\right\|_{L^{2}} .
$$

If $1 \leq l$, using Lemma 2.7 we have

$$
\begin{aligned}
\sum_{1 \leq l \leq k-1}\left\|\nabla^{l} f(n) \nabla^{k-l+1} u\right\|_{L^{2}}\left\|\nabla^{k+1} u\right\|_{L^{2}} & \lesssim \sqrt{\mathcal{E}_{0}^{3}}\left\|\nabla^{k+1}(n, u)\right\|_{L^{2}}\left\|\nabla^{k+1} u\right\|_{L^{2}} \\
& \lesssim \sqrt{\mathcal{E}_{0}^{3}}\left(\left\|\nabla^{k+1} n\right\|_{L^{2}}^{2}+\left\|\nabla^{k+1} u\right\|_{L^{2}}^{2}\right) .
\end{aligned}
$$

From (3.21), (3.22), and (3.23), we have

$$
\left|I_{2}\right| \lesssim \sqrt{\mathcal{E}_{0}^{3}}\left(\left\|\nabla^{k+1} n\right\|_{L^{2}}^{2}+\left\|\nabla^{k+1} u\right\|_{L^{2}}^{2}\right) .
$$

Now, we estimate the term $I_{3}$. Because $k \geq 1$, we can integrate by parts to have

$$
I_{3}=-\int_{\mathbb{R}^{3}} \nabla^{k}(g(n, q) \nabla n) \nabla^{k} u d x \leq\left\|\nabla^{k}(g(n, q) \nabla n)\right\|_{L^{\frac{6}{5}}}\left\|\nabla^{k} u\right\|_{L^{6}}
$$




$$
\begin{aligned}
& \leq \sum_{i=l}^{k}\left\|\nabla^{l} g(n, q) \nabla^{k+1-l} n\right\|_{L^{\frac{6}{5}}}\left\|\nabla^{k+1} u\right\|_{L^{2}}+\left\|g(n, q) \nabla^{k+1} n\right\|_{L^{\frac{6}{5}}}\left\|\nabla^{k+1} u\right\|_{L^{2}} \\
& :=\left(\sum_{i=1}^{k} I_{31}^{l}+I_{32}\right)\left\|\nabla^{k+1} u\right\|_{L^{2}} .
\end{aligned}
$$

For $I_{31}$, from the Leibniz formula we have

$$
\nabla^{l} g(n, q)=g^{r_{1}, \cdots, r_{m} ; \beta_{1}, \cdots, \beta_{k}}(n, q) \nabla^{r_{1}} n \cdots \nabla^{r_{m}} n \nabla^{\beta_{1}} q \cdots \nabla^{\beta_{h}} q,
$$

where

$$
r_{1}+\cdots+r_{m}+\beta_{1}+\cdots+\beta_{h}=l ; r_{i} \geq 1, \beta_{j} \geq 1,1 \leq i \leq m, 1 \leq j \leq h .
$$

Set $\alpha=\min \left\{r_{1}, \cdots, r_{m}, \beta_{1}, \cdots, \beta_{h}\right\}$; without loss of generality, we assume $r_{1}=\alpha$. It is obvious that $r_{1} \leq\left[\frac{k+1}{2}\right]$, so

$$
\begin{aligned}
& \left|I_{31}^{l}\right| \lesssim\left\|\nabla^{r_{1}} n\right\|_{L^{3}}\left\|\nabla^{r_{2}} n\right\|_{\frac{2\left(k+1-r_{1}\right)}{r_{2}}} \cdots\left\|\nabla^{r_{m}} n\right\|_{\frac{2\left(k+1-r_{1}\right)}{r_{m}}} \\
& \times\left\|\nabla^{\beta_{1}} q\right\|_{\frac{2\left(k+1-r_{1}\right)}{\beta_{1}}} \cdots\left\|\nabla^{\beta_{h}} q\right\|_{\frac{2\left(k+1-r_{1}\right)}{\beta_{h}}}\left\|\nabla^{k+1-r_{1}} n\right\|_{L} \frac{2\left(k+1-r_{1}\right)}{k+1-l} \\
& \lesssim\left\|\nabla^{r_{1}} n\right\|_{L^{3}}\|\nabla n\|_{L^{3}}{ }^{1-\frac{r_{2}}{k+1-r_{1}}}\left\|\nabla^{k+1-r_{1}} n\right\|_{L^{2}} \frac{r_{2}}{k+1-r_{1}} \\
& \times \cdots\|\nabla n\|_{L^{3}}{ }^{1-\frac{r_{m}}{k+1-r_{1}}}\left\|\nabla^{k+1-r_{1}} n\right\|_{L^{2}}{ }^{\frac{r_{m}}{k+1-r_{1}}} \\
& \times\|\nabla q\|_{L^{3}}{ }^{1-\frac{\beta_{1}}{k+1-r_{1}}}\left\|\nabla^{k+1-r_{1}} q\right\|_{L^{2}}{ }^{\frac{\beta_{1}}{k+1-r_{1}}}\|\nabla q\|_{L^{3}}{ }^{1-\frac{\beta_{h}}{k+1-r_{1}}}\left\|\nabla^{k+1-r_{1}} q\right\|_{L^{2}}{ }^{\frac{\beta_{h}}{k+1-r_{1}}} \\
& \lesssim\|\nabla(n, q)\|_{L^{3}}{ }^{k-r_{1}}\left\|\nabla^{r_{1}}(n, q)\right\|_{L^{3}}\left\|\nabla^{k+1-r_{1}}(n, q)\right\|_{L^{2}} \\
& \lesssim\left\|\nabla^{\alpha_{1}}(n, q)\right\|_{L^{2}}^{\theta}\left\|\nabla^{k+1}(n, q)\right\|_{L^{2}}^{1-\theta}\|(n, q)\|_{L^{2}}^{1-\theta}\left\|\nabla^{k+1}(n, q)\right\|_{L^{2}}^{\theta} \\
& \lesssim \sqrt{\mathcal{E}_{0}^{3}}\left\|\nabla^{k+1}(n, q)\right\|_{L^{2}}^{\theta}\left\|\nabla^{k+1}(n, q)\right\|_{L^{2}}^{1-\theta} .
\end{aligned}
$$

Here we use the Hölder inequality and Lemma 2.3, and $\alpha_{1}, \theta$ satisfy

$$
\left\{\begin{array}{l}
\frac{r_{1}}{3}-\frac{1}{3}=\left(\frac{\alpha_{1}}{3}-\frac{1}{2}\right) \theta+\left(\frac{k+1}{3}-\frac{1}{2}\right)(1-\theta), \\
\frac{k+1-r_{1}}{3}-\frac{1}{2}=\left(\frac{0}{3}-\frac{1}{2}\right)(1-\theta)+\left(\frac{k+1}{3}-\frac{1}{2}\right) \theta .
\end{array}\right.
$$

From (3.8),

$$
\theta=\frac{k+1-r_{1}}{k+1}, \quad \alpha=\frac{k+1}{2\left(k+1-r_{1}\right)} .
$$

So, $0<\theta<1, \alpha \in\left[\frac{1}{2}, 1\right)$.

For $I_{32}$, noticing that $g(n, q) \lesssim|n|$ we have

$$
\left|I_{32}\right| \lesssim\|n\|_{L^{3}}\left\|\nabla^{k+1}\right\|_{L^{2}} .
$$

From (3.25), (3.27), and (3.29), we have

$$
\left|I_{3}\right| \lesssim \sqrt{\mathcal{E}_{0}^{3}}\left\|\nabla^{k+1}(n, q)\right\|_{L^{2}}^{2} .
$$

For $I_{4}$, notice that $I_{4}$ is similar to $I_{3}$, the only difference being that the smooth function $g(n, q)$ is replaced by $h(n, q)$ with the same property. We have the following estimates:

$$
I_{4} \lesssim \sqrt{\mathcal{E}_{0}^{3}}\left\|\nabla^{k+1}(n, q)\right\|_{L^{2}}^{2}
$$


Finally, it remains to estimate the last term $I_{5}$.

$$
\begin{aligned}
& I_{5} \lesssim\left|\int_{\mathbb{R}^{3}} \Delta \nabla^{k} n \nabla^{k} \operatorname{div}(n u) d x\right| \lesssim\left\|\nabla^{k+2} n\right\|_{L^{2}}\left\|\nabla^{k+1}(n u)\right\|_{L^{2}} \\
& \lesssim \sum_{\ell=1}^{k}\left\|\nabla^{k+2} n\right\|_{L^{2}}\left\|\nabla^{\ell} n\right\|_{L^{4}}\left\|\nabla^{k+1-\ell} u\right\|_{L^{4}} \\
& +\left\|\nabla^{k+2} n\right\|_{L^{2}}\left(\|u\|_{L^{\infty}}\left\|\nabla^{k+1} n\right\|_{L^{2}}+\|n\|_{L^{\infty}}\left\|\nabla^{k+1} u\right\|_{L^{2}}\right) \\
& \lesssim M_{1}+M_{2} \text {. }
\end{aligned}
$$

For $M_{1}$,

$$
\begin{aligned}
M_{1} & =\sum_{\ell=1}^{k}\left\|\nabla^{k+2} n\right\|_{L^{2}}\left\|\nabla^{\ell} n\right\|_{L^{4}}\left\|\nabla^{k+1-\ell} u\right\|_{L^{4}} \\
& \lesssim \sum_{\ell=1}^{k}\left\|\nabla^{k+2} n\right\|_{L^{2}}\|n\|_{L^{\infty}}^{\theta}\left\|\nabla^{k+1} n\right\|_{L^{2}}^{1-\theta}\|u\|_{L^{\infty}}^{\theta}\left\|\nabla^{k+1} u\right\|_{L^{2}}^{1-\theta} \\
& \lesssim \epsilon\left\|\nabla^{k+1}(\nabla n, n, u)\right\|_{L^{2}}^{2} .
\end{aligned}
$$

where $\alpha$ is defined by

$$
\begin{gathered}
\frac{\ell}{3}-\frac{1}{4}=0 \times \theta+(1-\theta)\left(\frac{k+1}{3}-\frac{1}{2}\right), \\
\theta=\frac{4 \ell-3}{4(k+1)-6} \in(0,1) .
\end{gathered}
$$

For $M_{2}$,

$$
M_{2} \lesssim \epsilon\left\|\nabla^{k+1}(\nabla n, n, u)\right\|_{L^{2}}^{2} .
$$

From (3.33) and (3.34), we obtain

$$
I_{5} \lesssim \epsilon\left\|\nabla^{k+1}(\nabla n, n, u)\right\|_{L^{2}}^{2} .
$$

Finally, from (3.12), (3.13), (3.19), (3.24), (3.30), (3.31), and (3.35), choosing $\epsilon$ small enough, we conclude the Lemma 3.2.

Lemma 3.3. Under the assumption (3.3), for $k=0,1, \cdots, N$, there exist a positive constant $C$ satisfying

$$
\begin{aligned}
& \frac{1}{2} \frac{d}{d t} \int_{\mathbb{R}^{3}}\left|\nabla^{k} q\right|^{2} d x+C \int_{\mathbb{R}^{3}}\left|\nabla \nabla^{k} q\right|^{2} d x++\int_{\mathbb{R}^{3}} \nabla^{k} \operatorname{div} u \cdot \nabla^{k} q d x \\
\lesssim & \sqrt{\mathcal{E}_{0}^{3}}\left(\left\|\nabla^{k+1} n\right\|_{L^{2}}^{2}+\left\|\nabla^{k+2} n\right\|_{L^{2}}^{2}+\left\|\nabla^{k+1} q\right\|_{L^{2}}^{2}+\left\|\nabla^{k+1} u\right\|_{L^{2}}^{2}\right) .
\end{aligned}
$$

Proof. Applying $\nabla^{k}$ to $(3.1)_{3}$, multiplying $\nabla^{k} q$ and integrating by parts over $\mathbb{R}^{3}$

$$
\begin{aligned}
& \frac{1}{2} \frac{d}{d t} \int_{\mathbb{R}^{3}}\left(\nabla^{k} q\right)^{2} d x+\mu \int_{\mathbb{R}^{3}}\left(\nabla \nabla^{k} q\right)^{2} d x+\int_{\mathbb{R}^{3}} \nabla^{k} \operatorname{div} u \cdot \nabla^{k} q d x d x \\
= & -\int_{\mathbb{R}^{3}} \nabla^{k}(\nabla q \cdot u) \nabla^{k} q d x+\int_{\mathbb{R}^{3}} \nabla^{k}(f(n) \Delta q) \nabla^{k} q d x-\int_{\mathbb{R}^{3}} \nabla^{k}(B(n, q) \operatorname{div} u) \cdot \nabla^{k} q d x
\end{aligned}
$$




$$
\begin{aligned}
& \quad+\int_{\mathbb{R}^{3}} \nabla^{k}\left[\frac{1}{n+1}\left[2 \mu D(u): D(u)+\nu(\nabla u)^{2}\right] \nabla^{k} q d x+\int_{\mathbb{R}^{3}} \nabla^{k}\left[\frac{\mathcal{K}}{n+1}: \nabla u\right] \nabla^{k} q d x\right. \\
& \quad+\int_{\mathbb{R}^{3}} \nabla^{k}[\nabla \Delta n u] \nabla^{k} q d x \\
& =J_{1}+J_{2}+J_{3}+J_{4}+J_{5}+J_{6} .
\end{aligned}
$$

Applying the method used to estimate $I_{1}, I_{2}$, and $I_{3}$ in Lemma 3.2, we have the following estimates:

$$
\begin{aligned}
& \left|J_{1}\right| \leq \sqrt{\mathcal{E}_{0}^{3}}\left(\left\|\nabla^{k+1} q\right\|_{L^{2}}^{2}+\left\|\nabla^{k+1} u\right\|_{L^{2}}^{2}\right) . \\
& \left|J_{2}\right| \leq \sqrt{\mathcal{E}_{0}^{3}}\left\|\nabla^{k+1} q\right\|_{L^{2}}^{2} . \\
& \left|J_{3}\right| \leq \sqrt{\mathcal{E}_{0}^{3}}\left(\left\|\nabla^{k+1} q\right\|_{L^{2}}^{2}+\left\|\nabla^{k+1} u\right\|_{L^{2}}^{2}+\left\|\nabla^{k+1} q\right\|_{L^{2}}^{2}\right) .
\end{aligned}
$$

For $J_{4}$, integrating by parts over $\mathbb{R}^{3}$,

$$
\begin{aligned}
& \left|J_{4}\right| \leq\left|\int_{\mathbb{R}^{3}} \nabla^{k}\left[\frac{1}{n+1}\left[2 \mu D(u): D(u)+\nu(\nabla u)^{2}\right]\right] \nabla^{k} q d x\right| \lesssim\left|\int_{\mathbb{R}^{3}} \nabla^{k}\left[\frac{1}{n+1}(\nabla u)^{2}\right] \nabla^{k} q d x\right| \\
& \lesssim\left|\int_{\mathbb{R}^{3}} \nabla^{k}[t(n, \nabla n, u)(\nabla u)] \nabla^{k} q d x\right|+\left|\int_{\mathbb{R}^{3}} \nabla^{k}\left[m(n, u)\left(\nabla^{2} u\right)\right] \nabla^{k} q d x\right| \\
& +\left|\int_{\mathbb{R}^{3}} \nabla^{k}[m(n, u)(\nabla u)] \nabla^{k+1} q d x\right| \\
& =J_{41}+J_{42}+J_{43} \text {. }
\end{aligned}
$$

Similar to $I_{2}$, we have

$$
\left|J_{43}\right| \leq \sqrt{\mathcal{E}_{0}^{3}}\left(\left\|\nabla^{k+1} q\right\|_{L^{2}}^{2}+\left\|\nabla^{k+1} n\right\|_{L^{2}}^{2}+\left\|\nabla^{k+1} u\right\|_{L^{2}}^{2}\right) .
$$

Now, we turn to estimating $J_{41}$.

$$
\begin{aligned}
\left|J_{41}\right| & \lesssim\left|\int_{\mathbb{R}^{3}} \nabla^{k-1}[t(n, \nabla n, u)(\nabla u)] \nabla^{k+1} q d x\right| \lesssim \sum_{l=0}^{k-1}\left|\int_{\mathbb{R}^{3}} \nabla^{l} t(n, \nabla n, u) \nabla^{k-l} u \nabla^{k+1} q d x\right| \\
& \lesssim\left|\int_{\mathbb{R}^{3}} t(n, \nabla n, u) \nabla^{k} u \nabla^{k+1} q d x\right|+\sum_{l=1}^{k-1}\left|\int_{\mathbb{R}^{3}} \nabla^{l} t(n, \nabla n, u) \nabla^{k-l} u \nabla^{k+1} q d x\right| \\
& :=J_{411}+\sum_{l=1}^{k-1} J_{412}^{l} .
\end{aligned}
$$

For $J_{411}$, we have

$$
\left|J_{411}\right| \lesssim \sqrt{\mathcal{E}_{0}^{3}}\|\nabla n\|_{L^{3}}\left\|\nabla^{k} u\right\|_{L^{6}}\left\|\nabla^{k+1} q\right\|_{L^{2}} \lesssim \sqrt{\mathcal{E}_{0}^{3}}\left\|\nabla^{k+1}(u, q)\right\|_{L^{2}}^{2} .
$$

For $J_{412}^{l}$, using the Leibniz formula and noticing that $d_{1}+\cdots+d_{h}+c_{1}+\cdots+c_{m}=l+1$, we have

$$
\left|J_{412}^{l}\right| \lesssim\left\|\nabla^{d_{1}} n \cdots \nabla^{d_{h}} n \nabla^{c_{1}} u \cdots \nabla^{c_{m}} u \nabla^{k-l}\right\|_{L^{2}}\left\|\nabla^{k+1} q\right\|_{L^{2}}
$$


Using a method similar to that which proved Lemma 2.7, we have

$$
\left|J_{412}^{l}\right| \lesssim \sqrt{\mathcal{E}_{0}^{3}}\left\|\nabla^{k+1}(u, q)\right\|_{L^{2}}^{2} .
$$

Finally, from (3.44) and (3.46), we have

$$
\left|J_{41}\right| \lesssim \sqrt{\mathcal{E}_{0}^{3}}\left\|\nabla^{k+1}(u, q)\right\|_{L^{2}}^{2}
$$

Similar to $J_{41}$, we have

$$
\left|J_{42}\right| \lesssim \sqrt{\mathcal{E}_{0}^{3}}\left\|\nabla^{k+1}(u, q)\right\|_{L^{2}}^{2}
$$

From (3.48), and (3.42), (3.47) together with (3.41), we have

$$
\left|J_{4}\right| \leq \sqrt{\mathcal{E}_{0}^{3}}\left(\left\|\nabla^{k+1} n\right\|_{L^{2}}^{2}+\left\|\nabla^{k+2} n\right\|_{L^{2}}^{2}+\left\|\nabla^{k+1} q\right\|_{L^{2}}^{2}+\left\|\nabla^{k+1} u\right\|_{L^{2}}^{2}\right) .
$$

As for $J_{5}$, for $k \geq 1$, integrating by parts, we have

$$
\begin{aligned}
\left|I_{5}\right| & \leq \int_{\mathbb{R}^{3}} \nabla^{k}(\Delta n \mathcal{I}: \nabla u) \nabla^{k} q d x+\int_{\mathbb{R}^{3}} \nabla^{k}(w(n, \nabla n) \nabla u) \nabla^{k} q d x \\
& \lesssim\left\|\nabla^{k}(\Delta n \nabla u)\right\|_{L^{\frac{6}{5}}}\left\|\nabla^{k+1} q\right\|_{L^{2}}+\int_{\mathbb{R}^{3}} \nabla^{k}\left[\frac{1}{n+1}|\nabla n|^{2} \nabla u\right] \nabla^{k} q d x \\
& \lesssim\left\|\nabla^{k}\left(\nabla^{2} n \nabla u\right)\right\|_{L^{\frac{6}{5}}}\left\|\nabla^{k+1} q\right\|_{L^{2}}+\int_{\mathbb{R}^{3}} \nabla^{k}[w(n, \nabla n) \nabla u] \nabla^{k} q d x \\
& :=J_{51}+J_{52} .
\end{aligned}
$$

Using the method which bounded $W_{1}$ in Lemma 3.1, we can estimate

$$
\begin{aligned}
J_{51} & \lesssim \sqrt{\mathcal{E}_{0}^{3}}\left(\left\|\nabla^{k+2} n\right\|_{L^{2}}^{2}+\left\|\nabla^{k+1} u\right\|_{L^{2}}^{2}+\left\|\nabla^{k+1} q\right\|_{L^{2}}^{2}\right), \\
J_{52} & \lesssim \sqrt{\mathcal{E}_{0}^{3}}\left(\left\|\nabla^{k} \nabla n\right\|_{L^{2}}^{2}+\left\|\nabla^{k+1} u\right\|_{L^{2}}^{2}+\left\|\nabla^{k+1} q\right\|_{L^{2}}^{2}\right) .
\end{aligned}
$$

From (3.50), (3.51), and (3.52), we have

$$
J_{5} \lesssim \sqrt{\mathcal{E}_{0}^{3}}\left(\left\|\nabla^{k+1} n\right\|_{L^{2}}^{2}+\left\|\nabla^{k+2} n\right\|_{L^{2}}^{2}+\left\|\nabla^{k+1} q\right\|_{L^{2}}^{2}+\left\|\nabla^{k+1} u\right\|_{L^{2}}^{2}\right) .
$$

At last, we turn to estimate $J_{6}$. After integrating by parts, we have

$$
\begin{aligned}
J_{6} & \leq\left|\int_{\mathbb{R}^{3}} \nabla^{k}(\Delta n \operatorname{div} u) \nabla^{k} q d x\right|+\left|\int_{\mathbb{R}^{3}} \nabla^{k}(\Delta n u) \nabla^{k+1} q d x\right| \\
& \leq\left|\int_{\mathbb{R}^{3}} \nabla^{k}(\Delta n \operatorname{div} u) \nabla^{k} q d x\right|+\left|\int_{\mathbb{R}^{3}} \nabla^{k+1}(\nabla n u) \nabla^{k+1} q d x\right| \\
& :=J_{61}+J_{62} .
\end{aligned}
$$

$J_{61}$ is the same as $J_{51}$, and using Hölder's inequality and the method in (3.33), we have

$$
\begin{aligned}
J_{62} & \leq\left\|\nabla^{k+1}(\nabla n u)\right\|_{L^{2}}\left\|\nabla^{k+1} q\right\|_{L^{2}} \\
& \leq \sqrt{\mathcal{E}_{0}^{3}}\left(\left\|\nabla^{k+1} n\right\|_{L^{2}}^{2}+\left\|\nabla^{k+2} n\right\|_{L^{2}}^{2}+\left\|\nabla^{k+1}(u, q)\right\|_{L^{2}}^{2}\right) .
\end{aligned}
$$


Combining (3.51) and (3.55), we have

$$
J_{6} \lesssim \sqrt{\mathcal{E}_{0}^{3}}\left(\left\|\nabla^{k+1} n\right\|_{L^{2}}^{2}+\left\|\nabla^{k+2} n\right\|_{L^{2}}^{2}+\left\|\nabla^{k+1} q\right\|_{L^{2}}^{2}+\left\|\nabla^{k+1} u\right\|_{L^{2}}^{2}\right) .
$$

Finally, we conclude the Lemma 3.3 from (3.38), (3.39), (3.40), (3.49), (3.53), and (3.56).

The following lemma provides the dissipation estimate for $n$.

Lemma 3.4. Under the assumption (3.3), for $k=0,1, \cdots, N$, there exist positive constants $C_{1}$ and $C_{2}$ satisfying

$$
\frac{d}{d t} \int_{\mathbb{R}^{3}} \nabla^{k} u \cdot \nabla \nabla^{k} n d x+C_{1}\left(\left\|\nabla^{k+1} n\right\|_{L^{2}}^{2}+\left\|\nabla^{k+2} n\right\|_{L^{2}}^{2}\right) \leq C_{2}\left\|\nabla^{k+1} u\right\|_{L^{2}}^{2} .
$$

Proof. Applying $\nabla^{k}$ to $(3.1)_{2}$, multiplying $\nabla^{k} \nabla n$, integrating by parts over $\mathbb{R}^{3}$ and using Hölder's inequality,

$$
\begin{aligned}
& \int_{\mathbb{R}^{3}}\left|\nabla \nabla^{k} n\right|^{2} d x-\int_{\mathbb{R}^{3}} \nabla^{k} \nabla n \cdot \nabla \Delta \nabla^{k} n d x=\int_{\mathbb{R}^{3}}\left|\nabla \nabla^{k} n\right|^{2} d x+\int_{\mathbb{R}^{3}} \nabla^{k+2} n \cdot \nabla^{k+2} n d x \\
& \leq-\int_{\mathbb{R}^{3}} \nabla^{k} \partial_{t} u \cdot \nabla \nabla^{k} n d x+(2 \mu+|\lambda|)\left\|\nabla^{k+1} u\right\|_{L^{2}}\left\|\nabla^{k+2} n\right\|_{L^{2}} \\
& \quad+\int_{\mathbb{R}^{3}} \nabla^{k}(g(n, q) \nabla n+h(n, q) \nabla q) \cdot \nabla^{k+1} n d x \\
& \quad+\int_{\mathbb{R}^{3}} \nabla^{k}(u \cdot \nabla u+f(n)(\mu \Delta u+(\mu+\lambda) \nabla \operatorname{div} u u)) \cdot \nabla^{k+1} n d x .
\end{aligned}
$$

The delicate first term in the right hand side of (3.58) involves the time derivative, and the key idea is to integrate by parts in the $t$-variable and use the continuity equation. Thus by $(3.1)_{1}$ and integrating by parts for both the $t$ - and $x$-variables, we may compute

$$
\begin{aligned}
& -\int_{\mathbb{R}^{3}} \nabla^{k} u_{t} \cdot \nabla \nabla^{k} n d x \\
= & -\frac{d}{d t} \int_{\mathbb{R}^{3}} \nabla^{k} u \cdot \nabla \nabla^{k} n d x-\int_{\mathbb{R}^{3}} \nabla^{k} \operatorname{div} u \cdot \nabla^{k} n_{t} d x \\
= & -\frac{d}{d t} \int_{\mathbb{R}^{3}} \nabla^{k} u \cdot \nabla \nabla^{k} n d x+\left\|\nabla^{k} \operatorname{div} u\right\|_{L^{2}}^{2}+\int_{\mathbb{R}^{3}} \nabla^{k} \operatorname{div} u \cdot \nabla^{k} \operatorname{div} u(n u) d x .
\end{aligned}
$$

By Hölder's inequality, we have

$$
\int_{\mathbb{R}^{3}} \nabla^{k} \operatorname{div} u \cdot \nabla^{k} \operatorname{div}(n u) d x \lesssim\left\|\nabla^{k+1}(n u)\right\|_{L^{2}}\left\|\nabla^{k+1} u\right\|_{L^{2}} .
$$

By using the same method as in (3.35), we have

$$
\begin{aligned}
\left\|\nabla^{\ell} n \nabla^{k+1-\ell} u\right\|_{L^{2}} & \lesssim\left\|\nabla^{\ell} n\right\|_{L^{\infty}}\left\|\nabla^{k+1-\ell} u\right\|_{L^{2}} \\
& \lesssim\left\|\nabla^{\alpha} n\right\|_{L^{2}}^{1-\frac{\ell}{k+1}}\left\|\nabla^{k+1} n\right\|_{L^{2}}^{\frac{\ell}{k+1}}\|u\|_{L^{2}}^{\frac{\ell}{k+1}}\left\|\nabla^{k+1} u\right\|_{L^{2}}^{1-\frac{\ell}{k+1}} \\
& \lesssim \sqrt{\mathcal{E}_{0}^{3}}\left(\left\|\nabla^{k+1} n\right\|_{L^{2}}+\left\|\nabla^{k+1} u\right\|_{L^{2}}\right)
\end{aligned}
$$


where $\alpha$ is defined by

$$
\begin{aligned}
\frac{\ell}{3} & =\left(\frac{\alpha}{3}-\frac{1}{2}\right) \times\left(1-\frac{\ell}{k+1}\right)+\left(\frac{k+1}{3}-\frac{1}{2}\right) \times \frac{\ell}{k+1}, \\
& \Longrightarrow \alpha=\frac{3(k+1)}{2(k+1-\ell)} \leq 3 \text { because } \ell \leq \frac{k+2}{2} .
\end{aligned}
$$

While for $\ell>\left[\frac{k+1}{2}\right]+1$ (then $k+1-\ell \leq\left[\frac{k+1}{2}\right]$ ), we can then interchange the roles of $n$ and $u$ to deduce that (3.61) holds also for this case. Thus, in view of (3.59)-(3.61), we obtain

$$
-\int_{\mathbb{R}^{3}} \nabla^{k} u_{t} \cdot \nabla \nabla^{k} n d x \leq-\frac{d}{d t} \int_{\mathbb{R}^{3}} \nabla^{k} u \cdot \nabla \nabla^{k} n d x+C\left\|\nabla^{k+1} u\right\|_{L^{2}}^{2}+C \sqrt{\mathcal{E}_{0}^{3}}\left\|\nabla^{k+1} n\right\|_{L^{2}}^{2} .
$$

Applying the same method used in Lemma 2.7 and Lemma 3.1, we have

$$
\begin{aligned}
& \int_{\mathbb{R}^{3}} \nabla^{k}(u \cdot \nabla u+f(n)(\mu \Delta u+(\mu+\lambda) \nabla \operatorname{div} u)+g(n, q) \nabla n++h(n, q) \nabla q) \cdot \nabla^{k+1} n d x \\
\lesssim & \sqrt{\mathcal{E}_{0}^{3}}\left(\left\|\nabla^{k+1} n\right\|_{L^{2}}+\left\|\nabla^{k+1} u\right\|_{L^{2}}+\left\|\nabla^{k+1} q\right\|_{L^{2}}+\left\|\nabla^{k+2} n\right\|_{L^{2}}\right)
\end{aligned}
$$

Consequently, by (3.63), together with Cauchy's inequality, choosing $\sqrt{\mathcal{E}_{0}^{3}}<\epsilon$ small enough, we then complete the proof of Lemma 3.4.

\section{Negative Sobolev estimates}

In this section, our goal is to give some estimates of $\left(\Lambda^{-s} n, \Lambda^{-s} u, \Lambda^{-s} q, \Lambda^{-s} \nabla u\right)$. To control the nonlinear parts in (3.1), we need to use the the following $L^{p}$ type inequality for the Riesz potential. It can be found in [6, pp. 119]. In the sequel, we have to set $s \in\left(0, \frac{3}{2}\right)$.

If $\Lambda^{-s} f$ defined by (2.3) is the Riesz potential, then the Hardy-Littlewood-Sobolev theorem implies

$$
\left\|\Lambda^{-s} f\right\|_{L^{q}} \leq C\|f\|_{L^{p}}, \text { where } s \in(0,3), 1<p<q<\infty, \frac{1}{q}+\frac{s}{3}=\frac{1}{p} .
$$

We will establish the following lemma.

Lemma 4.1. Under the assumption (3.3), for $s \in(0,1 / 2]$ we have

$$
\begin{aligned}
& \frac{d}{d t} \int_{\mathbb{R}^{3}}\left|\Lambda^{-s} n\right|^{2}+\left|\Lambda^{-s} q\right|^{2}+\left|\Lambda^{-s} u\right|^{2}+\left|\Lambda^{-s} \nabla n\right|^{2} d x+C\left(\left\|\nabla \Lambda^{-s} u\right\|_{L^{2}}^{2}+C\left\|\nabla \Lambda^{-s} q\right\|_{L^{2}}^{2}\right) \\
\lesssim & \left(\|\nabla n\|_{H^{2}}^{2}+\|(\nabla u, \nabla q)\|_{H^{1}}^{2}\right)\left(\left\|\Lambda^{-s} n\right\|_{L^{2}}+\left\|\Lambda^{-s} q\right\|_{L^{2}}+\left\|\Lambda^{-s} u\right\|_{L^{2}}+\left\|\Lambda^{-s} \nabla n\right\|_{L^{2}}\right),
\end{aligned}
$$

and for $s \in(1 / 2,3 / 2)$ we have

$$
\begin{aligned}
& \quad \frac{d}{d t} \int_{\mathbb{R}^{3}}\left|\Lambda^{-s} n\right|^{2}+\left|\Lambda^{-s} q\right|^{2}+\left|\Lambda^{-s} u\right|^{2}+\left|\Lambda^{-s} \nabla n\right|^{2} d x+C\left(\left\|\nabla \Lambda^{-s} u\right\|_{L^{2}}^{2}+C\left\|\nabla \Lambda^{-s} q\right\|_{L^{2}}^{2}\right) \\
& \lesssim\|(n, u, q)\|_{L^{2}}^{s-1 / 2}\left(\|\nabla n\|_{H^{2}}+\|\nabla u\|_{H^{1}}+\|\nabla q\|_{H^{1}}\right)^{5 / 2-s} \\
& \quad \times\left(\left\|\Lambda^{-s} n\right\|_{L^{2}}+\left\|\Lambda^{-s}(u, q)\right\|_{L^{2}}+\left\|\Lambda^{-s} \nabla n\right\|_{L^{2}}\right) .
\end{aligned}
$$


Proof. Applying $\Lambda^{-s}$ to $(3.1)_{1},(3.1)_{2}$, and $(3.1)_{3}$ and multiplying the resulting identities by $\Lambda^{-s} n, \Lambda^{-s} u$, and $\Lambda^{-s} q$ respectively, summing up them, and then integrating over $\mathbb{R}^{3}$ by parts,

$$
\begin{aligned}
& \frac{1}{2} \frac{d}{d t} \int_{\mathbb{R}^{3}}\left|\Lambda^{-s}(n, u, q)\right|^{2} d x-\int_{\mathbb{R}^{3}} \Lambda^{-s} \nabla \Delta n \cdot \Lambda^{-s} u d x \\
& +\int_{\mathbb{R}^{3}} \mu\left|\nabla \Lambda^{-s} u\right|^{2}+(\mu+\lambda)\left|\operatorname{div} \Lambda^{-s} u\right|^{2} d x \\
= & \int_{\mathbb{R}^{3}} \Lambda^{-s}(-n \operatorname{div} u-u \nabla n) \Lambda^{-s} n-\Lambda^{-s}\left(u \nabla u+f(n)(\mu \Delta u+(\mu+\lambda) \nabla \operatorname{div} u) \Lambda^{-s} u d x\right. \\
& +\int_{\mathbb{R}^{3}} \Lambda^{-s}(g(n) \nabla n) \Lambda^{-s}+\int_{\mathbb{R}^{3}} \Lambda^{-s} \frac{\operatorname{div}(\mathcal{K} u)}{n+1} \Lambda^{-s} q d x u d x \\
& +\int_{\mathbb{R}^{3}} \Lambda^{-s}\left(-u \nabla q+f(n) \Delta q-B(n, q) \nabla u+\frac{1}{n+1}\left[2 \mu D(u): D(u)+\nu(\nabla u)^{2}\right]\right) \Lambda^{-s} q d x \\
:= & T_{1}+T_{2}+T_{3}+T_{4}+T_{5}+T_{7} .
\end{aligned}
$$

Let us treat $-\int_{\mathbb{R}^{3}} \Lambda^{-s} \nabla \Delta n \cdot \Lambda^{-s} u d x$ first.

$$
\begin{aligned}
& -\int_{\mathbb{R}^{3}} \Lambda^{-s} \nabla \Delta n \cdot \Lambda^{-s} u d x=\int_{\mathbb{R}^{3}} \Lambda^{-s} \Delta n \Lambda^{-s} \operatorname{div} u d x \\
= & \int_{\mathbb{R}^{3}}-\Lambda^{-s} \Delta n \Lambda^{-s} \partial_{t} n-\Lambda^{-s} \Delta n \Lambda^{-s} \operatorname{div}(n u) d x \\
= & \int_{\mathbb{R}^{3}}-\Lambda^{-s} \Delta n \Lambda^{-s} \partial_{t} \Delta n-\Lambda^{-s} \Delta n \cdot \Lambda^{-s} \operatorname{div} n u d x \\
= & \frac{1}{2} \frac{d}{d t} \int_{\mathbb{R}^{3}}\left|\Lambda^{-s} \nabla n\right|^{2} d x+\int_{\mathbb{R}^{3}} \Lambda^{-s} \nabla n \cdot \Lambda^{-s} \nabla^{2}(n u) d x . \\
:= & \frac{1}{2} \frac{d}{d t} \int_{\mathbb{R}^{3}}\left|\Lambda^{-s} \nabla n\right|^{2} d x+T_{6} .
\end{aligned}
$$

For $T_{1}$

$$
T_{1}=-\int_{\mathbb{R}^{3}} \Lambda^{-s}(n \operatorname{div} u) \Lambda^{-s} n d x \leq\left\|\Lambda^{-s}(n \operatorname{div} u)\right\|_{L^{2}}\left\|\Lambda^{-s} n\right\|_{L^{2}} .
$$

Applying inequality (4.1) to deal with $\left\|\Lambda^{-s}(n \operatorname{div} u)\right\|_{L^{2}}$, together with Hölder's inequality,

$$
\begin{aligned}
\left\|\Lambda^{-s}(n \operatorname{div} u)\right\|_{L^{2}} & \leq\|n \operatorname{div} u\|_{L^{1 / 2+s / 3}} \\
& \leq\|n\|_{L^{3 / s}}\|\nabla u\|_{L^{2}} \\
& \lesssim\|\nabla n\|_{L^{2}}^{1 / 2-s}\left\|\nabla^{2} n\right\|_{L^{2}}^{1 / 2+s}\|\nabla u\|_{L^{2}}\left\|\Lambda^{-s} n\right\|_{L^{2}} \\
& \lesssim\left(\|\nabla n\|_{H^{1}}^{2}+\|\nabla u\|_{L^{2}}^{2}\right)\left\|\Lambda^{-s} n\right\|_{L^{2}}
\end{aligned}
$$

From (4.6) and (4.7),

$$
T_{1} \leq\|n\|_{L^{3 / s}}\|\nabla u\|_{L^{2}}\left\|\Lambda^{-s} n\right\|_{L^{2}} .
$$

Similarly, we can bound the remaining terms by

$$
T_{2}=-\int_{\mathbb{R}^{3}} \Lambda^{-s}(u \cdot \nabla n) \Lambda^{-s} n d x \lesssim\|u\|_{L^{3 / s}}\|\nabla n\|_{L^{2}}\left\|\Lambda^{-s} n\right\|_{L^{2}},
$$




$$
\begin{aligned}
T_{3} & =-\int_{\mathbb{R}^{3}} \Lambda^{-s}(u \cdot \nabla u) \cdot \Lambda^{-s} u d x \lesssim\|u\|_{L^{3 / s}}\|\nabla u\|_{L^{2}}\left\|\Lambda^{-s} u\right\|_{L^{2}} \\
T_{4}= & -\int_{\mathbb{R}^{3}} \Lambda^{-s}(f(n, q)(\mu \Delta u+(\mu+\lambda) \nabla \operatorname{div} u)) \Lambda^{-s} u d x \\
& \lesssim\|(n, q)\|_{L^{3 / s}}\left\|\nabla^{2} u\right\|_{L^{2}}\left\|\Lambda^{-s} u\right\|_{L^{2}} \\
T_{5} & =-\int_{\mathbb{R}^{3}} \Lambda^{-s}(g(n, q) \nabla n) \cdot \Lambda^{-s} u d x \lesssim\|(n, q)\|_{L^{3 / s}}\|\nabla n\|_{L^{2}}\left\|\Lambda^{-s} u\right\|_{L^{2}} \\
T_{6} & =\int_{\mathbb{R}^{3}} \Lambda^{-s} \nabla n \cdot \Lambda^{-s} \nabla^{2}(n u) d x \leq\|(n, \nabla n, u)\|_{L^{3 / s}}\left\|\left(\nabla^{2} u, \nabla u, \nabla^{2} n\right)\right\|_{L^{2}}\left\|\Lambda^{-s} u\right\|_{L^{2}}
\end{aligned}
$$

$T_{7}$ contains many items, but the way of estimating each item in $T_{7}$ is similar to some $T_{i}(i=1, \cdots, 6)$.

$$
\left|T_{7}\right| \leq\|(n, \nabla n, u, q)\|_{L^{3 / s}}\left\|\left(\nabla^{2} u, \nabla u, \nabla^{2} n, \nabla q\right)\right\|_{L^{2}}\left\|\Lambda^{-s} q\right\|_{L^{2}} .
$$

So, from (4.8), (4.9), (4.10), (4.11), (4.12), (4.13), and (4.14), together with (4.4) and (4.5),

$$
\begin{aligned}
& \frac{1}{2} \frac{d}{d t} \int_{\mathbb{R}^{3}}\left|\Lambda^{-s} n\right|^{2}+\left|\Lambda^{-s} \nabla n\right|^{2}+\left|\Lambda^{-s} u\right|^{2}+\left|\Lambda^{-s} q\right|^{2} d x \\
& \quad+\int_{\mathbb{R}^{3}} \mu\left|\nabla \Lambda^{-s} u\right|^{2}+(\mu+\lambda)\left|\operatorname{div} \Lambda^{-s} u\right|^{2}+\left|\nabla \Lambda^{-s} q\right|^{2} d x \\
& \lesssim\|(n, u, \nabla n, q)\|_{L^{3 / s}}\|\nabla(n, u, q, \nabla u, \nabla n)\|_{L^{2}}\left\|\Lambda^{-s}(n, u, q, \nabla n)\right\|_{L^{2}} .
\end{aligned}
$$

Next, we turn to estimating $\|(n, u, q, \nabla n)\|_{L^{3 / s}}$.

Case 1. If $s \in\left(0, \frac{1}{2}\right]$, note that $\frac{3}{s} \geq 6$. To estimate $\|(n, \nabla n, u)\|_{L^{\frac{3}{s}}}$, a higher order of $(n, u)$ is needed:

$$
\begin{aligned}
\|u\|_{L^{\frac{3}{s}}} & \leq C\|\nabla u\|_{L^{2}}^{\frac{1}{2}-s}\left\|\nabla^{2} u\right\|_{L^{2}}^{\frac{1}{2}+\frac{s}{2}} \\
& \leq C\left(\|\nabla u\|_{L^{2}}+\left\|\nabla^{2} u\right\|_{L^{2}}\right) .
\end{aligned}
$$

So, the estimate of $\|(n, u, q \nabla n)\|_{L^{2}}$ is

$$
\|(n, u, q, \nabla n)\|_{L^{\frac{3}{s}}} \lesssim\|(\nabla n, \nabla u, \nabla q)\|_{H^{1}} .
$$

Combining (4.15) and (4.17), we conclude (4.2).

Case 2. If $s \in(1 / 2,3 / 2)$, note that $1 / 2+s / 3<1$ and $2<3 / s<6$. We will estimate $\|(n, u, \nabla n)\|_{L^{3 / s}}$ by interpolating between $L^{2}$ and $L^{6}$,

$$
\begin{aligned}
& \|u\|_{L^{\frac{3}{s}}} \leq\|u\|_{L^{2}}^{s-\frac{1}{2}}\|u\|_{L^{6}}^{\frac{3}{2}-s}, \\
& \|\nabla n\|_{L^{\frac{3}{s}}} \leq\|\nabla n\|_{L^{2}}^{s-\frac{1}{2}}\|\nabla n\|_{L^{6}}^{\frac{3}{2}-s}, \\
& \|n\|_{L^{\frac{3}{s}}} \leq\|n\|_{L^{2}}^{s-\frac{1}{2}}\|n\|_{L^{6}}^{\frac{3}{2}-s}, \\
& \|q\|_{L^{\frac{3}{s}}} \leq\|q\|_{L^{2}}^{s-\frac{1}{2}}\|q\|_{L^{6}}^{\frac{3}{2}-s} .
\end{aligned}
$$

So from (4.18), (4.19), (4.20), and (4.21),

$$
\|(n, u, q, \nabla n)\|_{L^{3 / s}} \lesssim\|(n, u, q, \nabla n)\|_{L^{2}}^{s-\frac{1}{2}}\|\nabla(n, u, q, \nabla n)\|_{L^{2}}^{\frac{3}{2}-s} .
$$

Consequently, from (4.15) and (4.22), we deduce Lemma 4.1. 


\section{The proof of the Theorem 1.1}

In this section, we shall combine Lemma 3.1, Lemma 3.1, Lemma 3.3, Lemma 3.4, Lemma 4.1, and the Sobolev interpolation to prove Theorem 1.1.

Summing up the estimates (3.5) of Lemma 3.1, (3.11) of Lemma 3.2, and (3.36) of Lemma 3.3 for each $k=0,1, \cdots, N$, because $\epsilon$ is small, we obtain

$$
\frac{d}{d t}\left(\left\|\nabla^{k} n\right\|_{L^{2}}^{2}+\left\|\nabla^{k} u\right\|_{L^{2}}^{2}+\left\|\nabla^{k} \nabla n\right\|_{L^{2}}^{2}+\left\|\nabla^{k} q\right\|_{L^{2}}^{2}\right)+C_{1}\left\|\nabla^{k+1} u\right\|_{L^{2}}^{2} \leq C_{2} \epsilon\left\|\nabla^{k+1} n\right\|_{H^{1}}^{2} .
$$

From (3.57) of Lemma 3.4,

$$
\frac{d}{d t} \int_{\mathbb{R}^{3}} \nabla^{k} u \cdot \nabla \nabla^{k} n d x+C_{3}\left\|\nabla^{k+1} n\right\|_{H^{1}}^{2} \leq C_{4}\left\|\nabla^{k} u\right\|_{H^{1}}^{2} .
$$

Multiplying (5.2) by $\delta / C_{4}$, adding with (5.1), choosing $\delta>0$ small enough, then there exists a constant $C_{5}>0$ satisfying

$$
\begin{aligned}
\frac{d}{d t}\left\{\left(\left\|\nabla^{k} n\right\|_{L^{2}}^{2}+\left\|\nabla^{k} u\right\|_{L^{2}}^{2}+\right.\right. & \left.\left.\left\|\nabla^{k} \nabla n\right\|_{L^{2}}^{2}+\left\|\nabla^{k} q\right\|_{L^{2}}^{2}\right)+\frac{\delta}{C_{4}} \int \nabla^{k} u \cdot \nabla \nabla^{k} n d x\right\} \\
& +C_{5}\left\{\left\|\nabla^{k+1} n\right\|_{H^{1}}^{2}+\left\|\nabla^{k+1} u\right\|_{L^{2}}^{2}+\left\|\nabla^{k+1} q\right\|_{L^{2}}^{2}\right\} \leq 0 .
\end{aligned}
$$

Denote $F^{k}(t)=\left\|\left(\nabla^{k} n, \nabla^{k} u, \nabla^{k} q, \nabla \nabla^{k} n\right)\right\|_{L^{2}}^{2}$, because $\delta$ is small enough, so that $F^{k}(t)$ is equivalent to the expression under the time derivative in (5.3). Then we may rewrite (5.3) as follows:

$$
\frac{d}{d t} F^{k}(t)+C_{5}\left\{\left\|\nabla^{k+1} n\right\|_{H^{1}}^{2}+\left\|\nabla^{k+1} u\right\|_{L^{2}}^{2}+\left\|\nabla^{k+1} q\right\|_{L^{2}}^{2}\right\} \leq 0 .
$$

Summing up (5.4) from $k=0$ to $k=N$, and then integrating directly in time, we get

$$
\|n(t)\|_{H^{N+1}}^{2}+\|u(t)\|_{H^{N}}^{2}+\|q(t)\|_{H^{N}}^{2} \lesssim \mathcal{E}_{0}^{3}(t) \leq \mathcal{E}_{0}^{3}(0) \lesssim\left\|n_{0}\right\|_{H^{N+1}}^{2}+\left\|u_{0}\right\|_{H^{N}}^{2}+\left\|q_{0}\right\|_{H^{N}}^{2} .
$$

This verifies (1.3).

Next, we turn to prove Theorem 1.1. Firstly, we need to verify that $\left\|\Lambda^{-s}(n, u, q, \nabla n)\right\|_{L^{2}} \leq C_{0}$ for all $t \geq 0$. By Lemma 4.1, we shall prove them for $s \in[0,1 / 2]$ first.

\section{Proof.}

Case 1. $s \in[0,1 / 2]$

Define $F_{-s}(t):=\left\|\Lambda^{-s} n(t)\right\|_{L^{2}}^{2}+\left\|\Lambda^{-s} u(t)\right\|_{L^{2}}^{2}+\left\|\Lambda^{-s} q(t)\right\|_{L^{2}}^{2}+\left\|\Lambda^{-s} \nabla n(t)\right\|_{L^{2}}^{2}$. Then, integrating (4.2) in time, by the bound (1.3) we obtain that, for $s \in(0,1 / 2]$,

$$
\begin{aligned}
F_{-s}(t) & \leq F_{-s}(0)+C \int_{0}^{t}\left(\|\nabla n\|_{H^{2}}^{2}+\|\nabla q\|_{H^{1}}^{2}+\|\nabla u\|_{H^{1}}^{2}\right) \sqrt{F_{-s}(\tau)} d \tau \\
& \leq C_{0}\left(1+\sup _{0 \leq \tau \leq t} \sqrt{F_{-s}(\tau)}\right) .
\end{aligned}
$$

This implies (1.5) for $s \in[0,1 / 2]$, that is,

$$
\left\|\Lambda^{-s} n(t)\right\|_{L^{2}}^{2}+\left\|\Lambda^{-s} u(t)\right\|_{L^{2}}^{2}+\left\|\Lambda^{-s} q(t)\right\|_{L^{2}}^{2}+\left\|\Lambda^{-s} \nabla n(t)\right\|_{L^{2}}^{2} \leq C_{0} \text { for } s \in[0,1 / 2] .
$$


If $\ell=1, \ldots, N$, we may use Lemma 2.6 to have

$$
\left\|\nabla^{\ell+1} f\right\|_{L^{2}} \geq C\left\|\Lambda^{-s} f\right\|_{L^{2}}^{-\frac{1}{\ell+s}}\left\|\nabla^{\ell} f\right\|_{L^{2}}^{1+\frac{1}{\ell+s}} .
$$

By this fact and (5.7), we find

$$
\left\|\nabla^{\ell+1} u\right\|_{L^{2}}^{2}+\left\|\nabla^{\ell+1} q\right\|_{L^{2}}^{2}+\left\|\nabla^{\ell+1} \nabla n\right\|_{L^{2}}^{2} \geq C_{0}\left(\left\|\nabla^{\ell} u\right\|_{L^{2}}^{2}+\left\|\nabla^{\ell} \nabla n\right\|_{L^{2}}^{2}\right)^{1+\frac{1}{\ell+s}} .
$$

This together with (1.3) implies in particular that for $k=0, \ldots, N$,

$$
\begin{gathered}
\left\|\nabla^{k+2} n\right\|_{L^{2}}^{2}+\left\|\nabla^{k+1} n\right\|_{L^{2}}^{2}+\left\|\nabla^{k+1} u\right\|_{L^{2}}^{2}+\left\|\nabla^{k+1} q\right\|_{L^{2}}^{2} \\
\geq C_{0}\left(\left\|\nabla^{k} n\right\|_{L^{2}}^{2}+\left\|\nabla^{k} u\right\|_{L^{2}}^{2}+\left\|\nabla^{k+1} n\right\|_{L^{2}}^{2}+\left\|\nabla^{k} q\right\|_{L^{2}}^{2}\right)^{1+\frac{1}{k+s}} .
\end{gathered}
$$

From (5.4) and (5.10), we obtain the following time differential inequality:

$$
\frac{d}{d t} F^{k}(t)+C_{0}\left(F^{k}(t)\right)^{1+\frac{1}{k+s}} \leq 0 \text { for } k=0, \ldots, N .
$$

Solving this inequality directly gives

$$
F^{k}(t) \leq C_{0}(1+t)^{-(k+s)} \text { for } k=0, \ldots, N .
$$

This implies that for $s \in[0,1 / 2]$, and $k=0, \ldots, N$,

$$
\left\|\nabla^{k} n(t)\right\|_{L^{2}}^{2}+\left\|\nabla^{k} u(t)\right\|_{L^{2}}^{2}+\left\|\nabla^{k} q(t)\right\|_{L^{2}}^{2}+\left\|\nabla^{k} \nabla n(t)\right\|_{L^{2}}^{2} \leq C_{0}(1+t)^{-(k+s)} .
$$

Case 2. $s \in\left(\frac{1}{2}, \frac{3}{2}\right)$.

Notice there is no damping effect on $n$, so the method for the case $s \in[0,1 / 2]$ can not be applied to this case. However, observing that we have $n_{0}, u_{0}, \nabla n_{0} \in \dot{H}^{-1 / 2}$ because $\dot{H}^{-s} \cap L^{2} \subset \dot{H}^{-s^{\prime}}$ for any $s^{\prime} \in[0, s]$, we then deduce from what we have proved for Theorem 1.1 with $s=1 / 2$ that the following decay result holds for $k=0, \ldots, N$ :

$$
\left\|\nabla^{k} n(t)\right\|_{L^{2}}^{2}+\left\|\nabla^{k} q(t)\right\|_{L^{2}}^{2}+\left\|\nabla^{k} u(t)\right\|_{L^{2}}^{2}+\left\|\nabla^{k} \nabla n(t)\right\|_{L^{2}}^{2} \leq C_{0}(1+t)^{-(k+1 / 2)} .
$$

Hence, by (5.14), we deduce from (4.3) that, for $k \in(1 / 2,3 / 2)$,

$$
\begin{aligned}
F_{-s}(t) & \leq F_{-s}(0)+C \int_{0}^{t}\|(n, u, q)\|_{L^{2}}^{s-1 / 2}\left(\|n\|_{H^{2}}+\|\nabla u\|_{H^{1}}+\|\nabla q\|_{H^{1}}\right)^{5 / 2-s} \sqrt{F_{-s}(\tau)} d \tau \\
& \leq C_{0}+C_{0} \int_{0}^{t}(1+\tau)^{-(7 / 4-s / 2)} d \tau \sup _{0 \leq \tau \leq t} \sqrt{F_{-s}(\tau)} \\
& \leq C_{0}\left(1+\sup _{0 \leq \tau \leq t} \sqrt{F_{-s}(\tau)}\right) .
\end{aligned}
$$

This implies (1.5) for $s \in(1 / 2,3 / 2)$, that is,

$$
\left\|\Lambda^{-s} n(t)\right\|_{L^{2}}^{2}+\left\|\Lambda^{-s} u(t)\right\|_{L^{2}}^{2}+\left\|\Lambda^{-s} q(t)\right\|_{L^{2}}^{2}+\left\|\Lambda^{-s} \nabla n(t)\right\|_{L^{2}}^{2} \leq C_{0} \quad \text { for } s \in(1 / 2,3 / 2) .
$$

Now that we have proved (5.16), we may repeat the arguments leading to Theorem 1.1 for $s \in[0,1 / 2]$ to prove that they hold also for $s \in(1 / 2,3 / 2)$.

Acknowledgment. This work is supported by National Natural Science Foundation of China-NSF (Grant No. 11271305). 


\section{REFERENCES}

[1] A. Matsumura and T. Nishida, The initial value problem for the equations of motion of compressible viscous and heat-conductive fluids, Proc. Japan Acad. Ser. A., 55, 337-342, 1979.

[2] A. Matsumura and T. Nishida, The initial value problems for the equations of motion of viscous and heat-conductive gases, J. Math. Kyoto Univ., 20, 67-104, 1980.

[3] B. Haspot, Existence of global weak solution for compressible fluid models of Korteweg type, J. Math. Fluid Mech., 13, 223-249, 2011.

[4] D.J. Korteweg, Sur la forme que prennent les équations du mouvement des fluides si l'on tient compte des forces capillaires causées par des variations de densité considérables mais continues et sur la théorie de la capillarité dans l'hypothèse d'une variation continue de la densité, Archives Néerlandaises de Sciences Exactes et Naturelles, 1-24, 1901.

[5] D. Bresch, B. Desjardins, and C.K. Lin, On some compressible fluid models: Korteweg, lubrication and shallow water system, Commun. Part. Diff. Eqs., 28, 843-868, 2003.

[6] E.M. Stein, Singular Integrals and Differentiability Properties of Functions, Princeton University Press, 1970.

[7] J.E. Dunn and J. Serrin, On the thermomechanics of interstitial working, Arch. Rat. Mech. Anal., 88, 95-133, 1985.

[8] H.L. Li, A. Matsumura, and G. Zhang, Optimal decay rate of the compressible Navier-StokesPoisson system in $\mathbb{R}^{3}$, Arch. Rat. Mech. Anal., 196, 681-713, 2010.

[9] H. Bahouri, J.Y. Chemin, and R. Danchin, Fourier Analysis and Nonlinear Partial Differential Equations, Springer-Verlag, 2011

[10] H. Hattori and D. Li, Solutions for two dimensional system for materials of Korteweg type, SIAM J. Math. Anal., 25, 85-98, 1994.

[11] H. Hattori and D. Li, Global solutions of a high dimensional system for Korteweg materials, J. Math. Anal. Appl., 198, 84-97, 1996.

[12] L. Nirenberg, On elliptic partial differential equations, Ann. Scuola Norm. Sup. Pisa, 13, 115$162,1959$.

[13] R. Danchin and B. Desjardins, Existence of solutions for compressible fluid models of Korteweg type, Ann. Inst. H. Poincar Anal. Non Linaire, 18, 97-133, 2001.

[14] Z. Tan and Y. Wang, Global solution and large-time behavior of the 3D compressible Euler equations with damping, J. Diff. Eqs., 254, 1686-1704, 2013.

[15] Y.J. Wang, Decay of the Navier-Stokes-Poisson equations, J. Diff. eqs., 253, 273-297, 2012.

[16] M. Kotschote, Strong solutions for a compressible fluid model of Korteweg type, Ann. Inst. H. Poincar-Anal. Non Lin., 25, 679-696, 2008.

[17] Y.J. Wang and Z. Tan, Optimal decay rates for the compressible fluid models of Korteweg type, J. Math. Anal. Appl., 379, 256-271, 2011.

[18] Y.N. Li, Global existence and optimal decay rate of the compressible Navier-Stokes-Korteweg equations with external force, J. Math. Anal. Appl., 388, 1218-1232, 2012.

[19] Y. Wang and Z. Tan, Global existence and optimal decay rate for the strong solutions in $H^{2}$ to the compressible Navier-Stokes equations, Appl. Math. Lett., 24, 1778-1784, 2011.

[20] Z. Tan and Y. Wang, Large time behavior of solutions to the isentropic compressible fluid models of Korteweg type in $R^{3}$, Commun. Math. Sci., 10, 1207-1223, 2012.

[21] Z. Tan and G.C. Wu, Global existence for the non-isentropic compressible Navier-StokesPoisson system in three and higher dimensions, Nonlinear Anal. Real World Appl., 13, 650-664, 2012.

$[22]$ Z. Tan, H.Q. Wang, and J.K. Xu, Global existence and optimal $L^{2}$ decay rate for the strong solutions to the compressible fluid models of Korteweg type, J. Math. Anal. Appl., 390, 181-187, 2012. 